\title{
Development of the control system for the VisLab Intercontinental Autonomous Challenge
}

\author{
Alberto Broggi, Paolo Medici, Elena Cardarelli, \\ Pietro Cerri, Alessandro Giacomazzo \\ VisLab - Dipartimento di Ingegneria dell'Informazione \\ Università degli Studi di Parma \\ $\{$ broggi, medici, cardar, cerri,giacom\}@vislab. it
}

\author{
Nicola Finardi \\ Tierra - Telematic Division \\ Topcon Positioning System \\ nfinardietopcon.com
}

\begin{abstract}
This paper presents the control system of an autonomous vehicle capable of perceiving and describing the environment using different inputs, such as GPS waypoints, roadways borders and lines, leader vehicles, and obstacles to be avoided. The controller has been implemented and tested for the VisLab Intercontinental Autonomous Challenge, a long intercontinental trip that aims to demonstrate capabilities of modern autonomous vehicles. To fulfill this mission a generalpurpose real-time motion planning system was designed and implemented. This pathplanner, based on the estimation of feasible trajectories on a cost map, is described and analyzed. System perfomance has been evaluated during tests: experimental results have demonstrated the capability of the system in vehicle following.
\end{abstract}

\section{INTRODUCTION}

Since the theme of the World Expo 2010, held in Shanghai, China, is 'better cities, better life', VisLab prepared an expedition focused on sustainable mobility. The challenge, named 'VisLab Intercontinental Autonomous Challenge (VIAC)', has a unique final goal: to design vehicles able to drive autonomously along a $13,000 \mathrm{~km}$ trip, with no human intervention. Although this goal is definitely very complex, VisLab approached this exciting endeavor together with additional innovative ideas. The vehicles are electric and power is partially delivered by solar panels. These additional requirements, if the challenge will be won, will help to demonstrate that it is possible - although in a prototype version - to move goods between two continents with nonpolluting vehicles powered by green energy and with virtually no human intervention.

This paper address these challenges by presenting the vehicle control system.

In recent years, several milestones have been set of autonomous driving, and several of these showed the VisLab research group at the forefront, like in the ARGO project [1] (a passenger car that in 1998 drove for 2000+ km on Italian highways $94 \%$ in automatic mode), or the TerraMax vehicle, an Oshkosh MTVR truck that VisLab equipped with sensing systems (primarily artificial vision) and that was able to reach the end of the DARPA Grand Challenge in 2005 (220 miles of off-road driving with no human intervention) and was qualified for the DARPA Urban Challenge in 2007 (6 hours of urban driving).
Team Caltech's Alice, Stanford's Stanley [2], Carnegie Mellon's Boss and MIT's Talos are other examples of stateof-the-art of autonomous driving in real environment (OffRoad and Urban). They have been fielded and demonstrated in challenges defined by clear rules and unfriendly environments. In our experiments, on the other a hand, it is not possible to exploit a priori knowledge of the world.

Representing, building, storing, and evaluating the possible trajectories have been a major thrust of research in robots motion planning. The TerraMax [3] in the Urban Challenge implemented different state-of-art trajectory generators according to a high level behavior supervisor: depending on the tasks that must be executed, and environmental conditions, one of four trajectory planners are selected.

Alice [4] implements generation of optimal trajectories using a nonlinear optimization technique of cost function: the initial rough trajectories are optimized at a later stage. After a trajectory is generated, it is followed by a lateral control error tracking.

With a similar approach Stanley [5] presented a new manner of tracking the generated trajectory based on an estimation of the lateral error and considering the front wheels orientation.

Boss [6] uses a model-predictive trajectory generator, same as one proposed by Howard and Kelly [7], to produce dynamically feasible actions between initial and desidered vehicle states using numerical linearization and inversion of the forward vehicle dynamic model.

Talos [8] implemented a variant of RTT, a widespread approach to select the most promising trajectories. The feasibility of the proposed trajectory are evaluated on a drivability grid, where each cell stores a drivable/non-drivable flag and the cost to drive over it.

All these robots show a clear division between the path planner and the low level steering control, having to perform global tasks which require a medium-term planning. In fact they demonstrated that the use of a global planner allows to execute complex maneuvers as Parking and U-Turns.

To accomplish the VIAC mission, the vehicle will be able to run using a local planning, but it must be also capable extended in the future to a global planner version. Methods based on potential field to evaluate trajectory are common in the literature, allowing a modular development of sensors. 
For this reason the trajectory is generated from the evaluation on a cost map of a subset of possible vehicle's eligible trajectories. An optimal trajectory is provided at a high ratio in order to cope with the unstable nature of vehicle dynamics.

All these works have shown that the proper knowledge of vehicle dynamics is a must. For this reason a calibration phase is also described in this paper, which allows to generate the most reliable trajectories as well. Finally, the results on the preliminary testing stages are given, based on a number of kilometers of automatic driving.

\section{The VeHicles}

Thanks to the cooperation with Piaggio, the vehicles selected for VIAC are Piaggio Porter Electric Power (Figure 1).

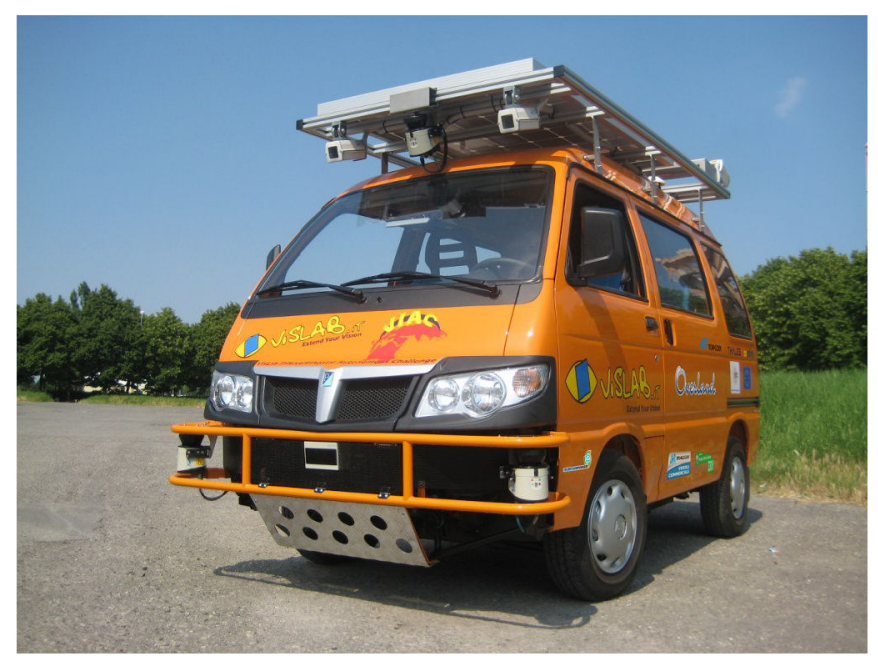

Fig. 1. The equipped vehicle used in VIAC.

The control system takes advantage of information provided by the TopCon AGI3, an inertial and geolocation device, installed on the vehicle roof. The GPS can use the Egnos/WAAS correction in order to achieve sub-meter accuracy, and gyroscopes show a noise level less than $0.03 \mathrm{rad} / \mathrm{sec}$ at working temperature. Both provide their information at $20 \mathrm{~Hz}$ rate.

As far as the actuation part is concerned, the design imposed that all servos had to be easily overridden by any user.

1) A servo motor, provided again by TopCon, is directly connected to the steering wheel column and controlled by position, speed, and torque through messages on the CAN-BUS. Internally the set-point is followed using a PID controller.

2) As the vehicle is electric, the speed is adjusted by changing the duty cycle of the PWM that controls the engine.

3) The brake system is implemented by an electric motor which acts directly on the braking pedal.

The prototype is equipped with five LIDARs and seven digital cameras: this sensory system makes the vehicle capable of frontal, lateral and rear sensing.

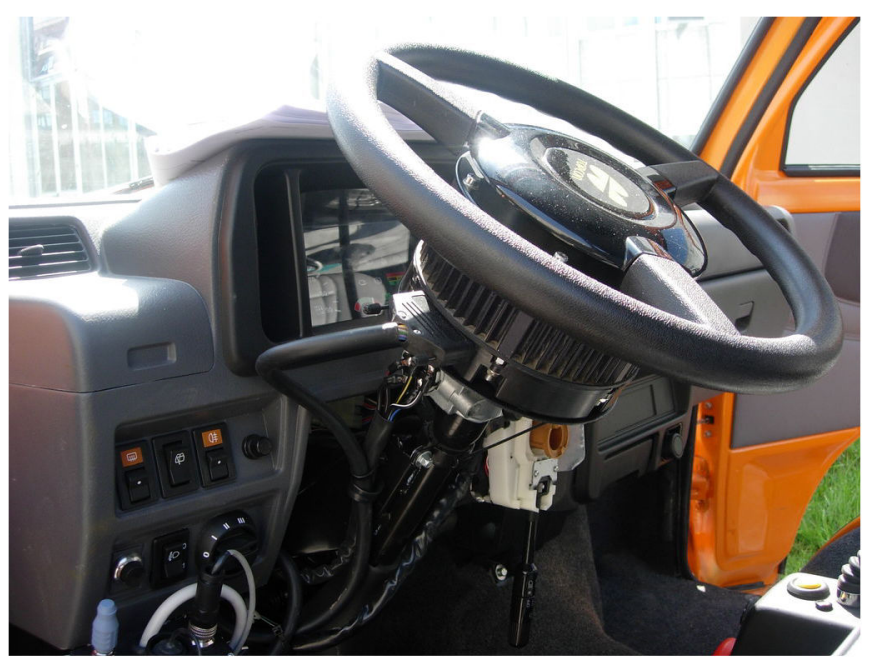

Fig. 2. The by-wire steering system.

The processing units are composed by 3 off-the-shelf multi-core computers, connected by gigaethernet, two of which are dedicated to sensing and one devoted entirely to planning and control.

\section{Kinematic Model}

A car can be viewed as an orientable point moving in the plane. As it commonly appears in the literature, this point in the kinematic model is located on the rear axle. Its configuration is defined by position $(x, y)$ and orientation $\theta$ in an absolute reference frame.

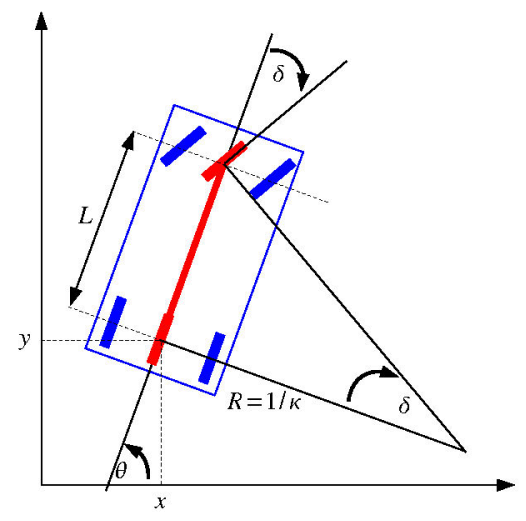

Fig. 3. Kinematic model of the vehicle in the Cartesian plane.

The model of the system motion (shown in fig. 3) is described by the kinematic bicycle differential equations:

$$
\begin{aligned}
& \dot{x}=\cos \theta \\
& \dot{y}=\sin \theta \\
& \dot{\theta}=\kappa \\
& \dot{\kappa}=\sigma
\end{aligned}
$$

where $\kappa$ is the curvature and $\sigma$ the sharpness of the followed path. The notation $\dot{f}$ indicates the derivative with respect to the drive distance. Typically the steering control is faster than 
the variation of longitudinal velocity, and the speed can be considered constant.

The longitudinal velocity of the car is denoted by $v$ and the curvature change velocity is given by $\sigma$. This model assumes that the wheels do not skid and, at the maximum vehicle speed, it was experimentally verified that this contribution is negligible.

To have realistic motion simulation, the maximum steering angle and the steering wheel turning speed are bounded according to:

$$
|\kappa|<\kappa_{\max }, \quad|\sigma|<\sigma_{\max }
$$

where $\kappa_{\max }$ and $\sigma_{\max }$ are experimentally measured.

The relation between steering angle and curvature path is given by the Ackermann kinematic model:

$$
\kappa=\frac{\tan \delta}{L}
$$

where $\delta$ is the front wheel angle (i.e. the angle between wheel and longitudinal axis of the car), and $L$ is the distance in meters between the front and rear axles.

Finally it is assumed that the relationship between the angle of the front wheels $\delta$ and the steering wheel angle $\phi$ is linear and time invariant:

$$
\delta=\alpha \phi
$$

According to Ackermann kinematics, maintaining a constant steering wheel angle, the vehicle follows circular trajectories. It is important to link the steering angle (the input control) to the curvature that the vehicle will perform.

\section{A. Model parameters recovery}

To achieve this, it is convenient to derive the model parameters by directly observing the vehicle reactions, measuring the radius of curvature produced by a particular steering angle. To measure the instantaneous curvature of the vehicle, various techniques are available. Due to low noise, the technique based on yaw-rate is preferable. In fact, instantaneous curvature is

$$
\kappa=\frac{r}{v}
$$

where $r$ is the yaw-rate provided by the on-board gyroscope. Comparing the curvature values obtained from equation 5 with the ones predicted by equation 3, using an optimization method, it is possible to recover the model parameters that minimize the error.

The results obtained after error minimization are presented, evaluating the correspondence between the curvature predicted by steering and that measured by the gyroscope: as shown in figure 4 , the predicted curvature involves less noisy contributions than the inertial one.

\section{B. Steer Offset estimation}

The electrical power steering wheel lacks an encoder that stores the zero position before switching off.

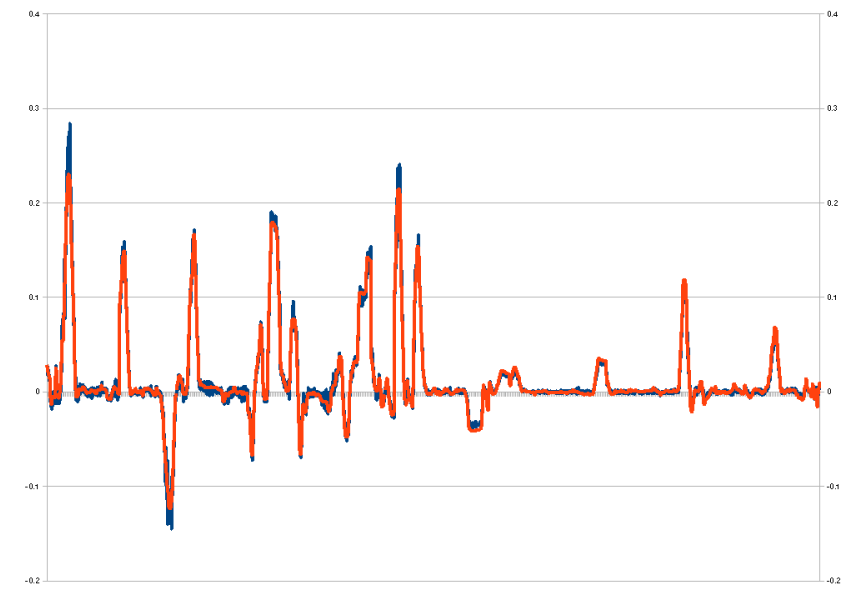

Fig. 4. Curvature: blue measured using inertial information in equation 5, and red predicted by steering using equation 3 .

For this reason in the first phase of the automatic driving (bootstrap), the vehicle reacts to small biases of yaw-rate and the steering wheel offset minimizing the error

$$
\phi-\phi_{0} \approx \frac{L r}{\alpha \nu}
$$

and returning the best value for $\phi_{0}$.

\section{Controller Design}

In order to provide reliability in steering, throttle, and brake control, the pathplanner computes different inputs obtained by camera and laser processing, GPS/INS, and high level control interfaces, as shown in figure 5 . The kinematic model is defined according to these information: it allows to provide the feasible speed $v$ and the curvature $\kappa$ that define the set point the control aims to reach.

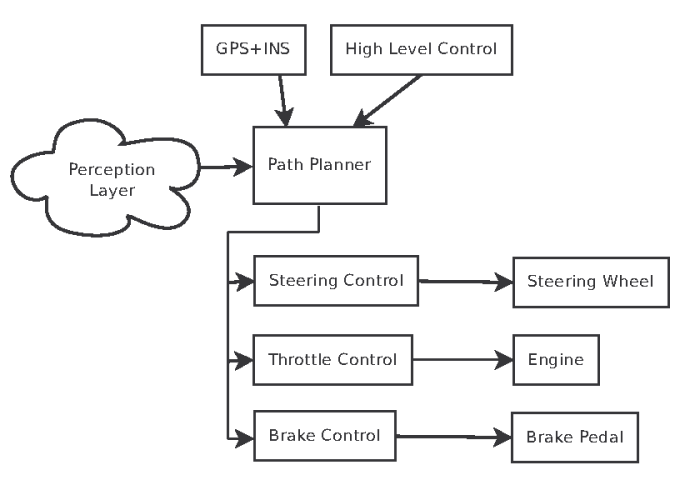

Fig. 5. Flowchart of the Planning and Control Layer.

\section{A. Cost maps}

Perception data processing introduces a delay between data acquisition on time $t_{0}$ and coordinates information availability on planning time $t_{1}$ : this means that the information 


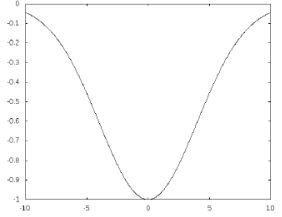

(a)

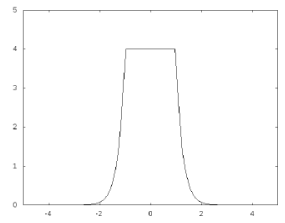

(c)

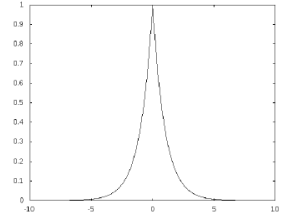

(b)

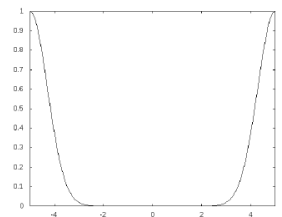

(d)
Fig. 6. Ideal primitives for cost map: (a) Waypoints. (b) Lanes. (c) Obstacles. (d) Ditches.

about the perceived vehicle coordinates does not refer to the current time $t_{1}$, but it is relative to the data at time $t_{0}$. To cope with this problem, the points referred to $t_{0}$ are rototranslated, according to vehicle dynamics, in order to guess their position on $t_{1}$.

Since GPS waypoints information, unlike perception data, are not represented in vehicle coordinates, but in world coordinates, conversion to time $t_{1}$ is directly performed. We decide to consider vehicle coordinates because when GPS information are not available, perception data could not be converted into world coordinates. Moreover vehicle coordinates are more accurate then GPS ones, although they have the typical problems of drift.

According to the input information, a cost map is generated in order to determine the feasible areas. Using a cost map it is possible to handle different inputs in a unified way and at the same time implement complex behaviors [9]. Figure 6 shows the ideal primitives used to generate the map according to the type of input: waypoints, lanes, obstacles and ditches (i.e. 'ditches' here refer to negative obstacles on the road side). Each input has a different weight according to its importance: obstacles and ditches are dangerous, then their weight is greater than the lanes or waypoints ones. Despite less accuracy, caused by input information quantization, cost maps provide more scalable and computationally efficient solutions than other approaches.

An occupancy grid, $280 \times 280$ pixels large representing $28 \times 28$ meters, is used. Each cell stores a floating point number encoding road conditions of the area surrounding the vehicle: low values represent safe regions, whereas high values are used to locate dangerous ones. A $10 \mathrm{~cm}$ cell size demonstrates to fit our need for a reliable and accurate navigation.

\section{B. Trajectory Planner}

When the curvature is constant the vehicle follows circular trajectories, but while the vehicle's wheel is turned, it introduces a curvature variation that is not negligible, so during changes of curvature the vehicle's motion is estimated with

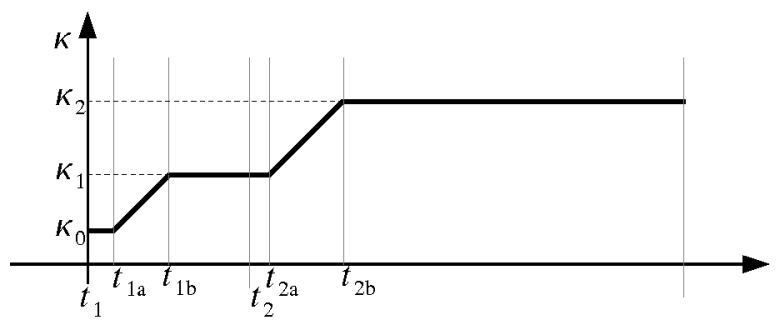

Fig. 7. Variation of the curvature function of time. Two similar simulations steps are performed to generate an acceptable trajectory.

a clothoid.

To perform steering control, all feasible trajectories are evaluated in order to determine, using cost maps, the best one to follow. The implemented planner reschedules the best trajectory at fixed times (time scheduling has been chosen to be $100 \mathrm{~ms}$ ). In the planning phase, all possible curvatures $\kappa_{1} \in\left[\kappa_{0}-\kappa_{l}, \kappa_{0}+\kappa_{l}\right]$ are considered, where $\kappa_{0}$ is the currently performed curvature and $\kappa_{l}$ is the maximum curvature variation that the vehicle is able to execute in the control time range. Considering that According to equations 3 and 5, the current curvature is estimated as:

$$
\kappa_{0}= \begin{cases}\frac{\tan \phi}{L} & v<v_{\gamma} \\ \frac{r}{v} & v>v_{\gamma}\end{cases}
$$

because at low speeds steering angle is more feasible than yaw rate, but at high speed yaw-rate can cope with the realistic vehicle motion (for examples bank rolls, drifts or wind). Currently the specified threshold $v_{\gamma}$ is $10 \mathrm{~km} / \mathrm{h}$.

For each evaluated path the maximum curvature depends on both joint limits, as the maximum steering angle $\kappa_{\text {max }}$, and comfort constraints, as the lateral acceleration $|\kappa|<a_{\max } / v^{2}$, defined to prevent fishtailing.

For each curvature $\kappa_{1}$, the corresponding trajectory is estimated in order to evaluate the best path that define, at $t_{1}$, the new steering set-point. Figure 7 shows the curvature variation with respect to the time:

- from $t_{1}$ to $t_{1 a}$ the vehicle does not react to the new setpoint and it keeps the curvilinear trend $\kappa_{0}$;

- from $t_{1 a}$ to $t_{1 b}$ the curvature changes from $\kappa_{0}$ to $\kappa_{1}$ following a clothoid;

- between $t_{1 b}$ and $t_{2}$ the vehicles follows the curvilinear trend $\kappa_{1}$.

In the same way on $t_{2}$ the vehicle will execute a new replanning: again for each evaluated $\kappa_{1}$, new values $\kappa_{2}$ in a neighborhood of $\kappa_{1}$ are considered and the trajectory for the next 8 meters is estimated. In such way the lookahead distance is partially speed-dependent and ranges from 8 to 11 meters.

Examples of trajectories generated during a curve and the lower cost path associated with the chosen route are shown in figure 8.

For each path the integral of the cost map is computed: this allows to find the future set-point as the $\kappa_{1}$ relative to the trajectory with the minimum integral. However vehicles dimensions must be considered when trajectory cost is evaluated: as shown in Figure 9, six distinctive points (instead of 


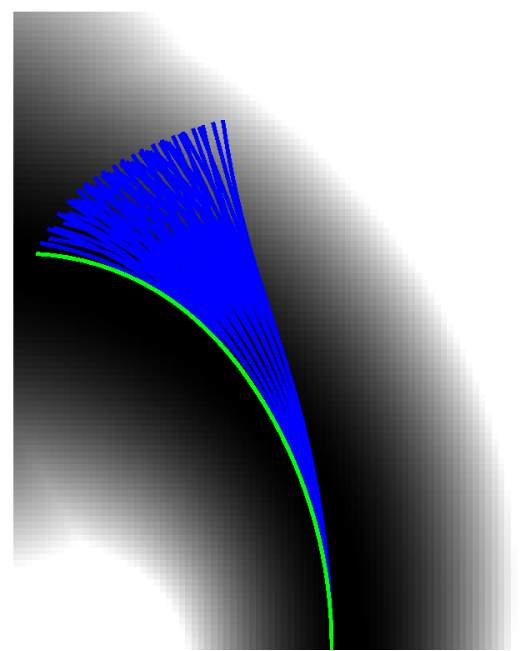

Fig. 8. Evaluation of the vehicle trajectories during a sharp curve: blue curves are evaluated trajectories, while the green one is the best. In the potential map dark areas represent points at low cost, while the light areas are high cost points.

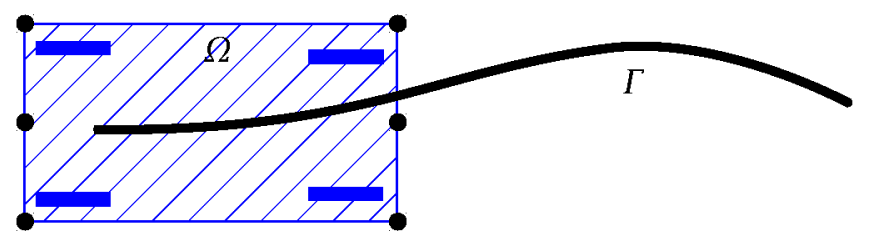

Fig. 9. Model used to consider vehicle dimensions for obstacle avoidance.

whole area $\Omega$ ) are considered in order to estimate, for each step, the maximum of cost map on the vehicle's covered area.

A fixed sampling step is used for $k_{1}$ and $k_{2}$, with the result of evaluate, at low speeds, a greater number of trajectories than with those of high speeds. The sampling step for $k_{1}$ is $0.0015 \mathrm{rad} / \mathrm{m}$, and it is further increased at the second simulation step to $0.01 \mathrm{rad} / \mathrm{m}$. Overall 917 configurations are on average computed, with peaks of about 16000 .

Exhaustive analysis of all trajectories is performed: to reduce computation overhead caused by huge number of trajectories to process, the sampling method described in [7] could be applied. It will provide a fast numerical technique to optimize the choice of trajectories focusing only on the interested ones.

In order to avoid unnecessary changes of direction, if the new curvature $\kappa_{1}$ is in a neighborhood of $5 \%$ near the past one, previous trajectory is kept.

Finally, this set-point is converted in steering wheel angle $\phi$ and transmitted to the steering control.

Since the path is constantly monitored, the planner adjusts the controller signal so that the vehicle can achieve the best path even in the presence of noise (for example moving obstacles) and track errors.

\section{Speed Control}

Current speed, acceleration (deceleration) are computed according to different contributes:

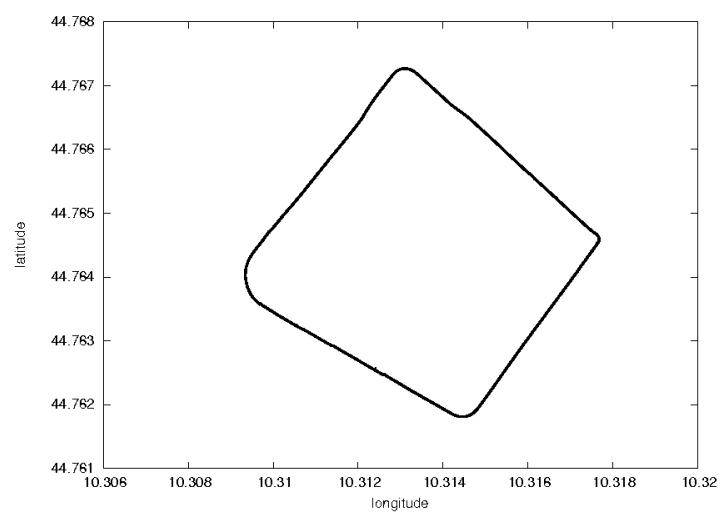

Fig. 10. The test-bed: University of Parma campus area.

- The speed set by high level control interfaces.

- The road roughntess, evaluated considering the INS information.

- The lateral acceleration in order to ensure stability avoiding vehicle skid; the speed bounded by the lateral acceleration is defined by:

$$
v^{2}<\frac{a_{y_{\max }}}{|\kappa|}
$$

- A target speed $v_{k}$, that allows to keep a security distance $d$ with the front vehicle in Leader Follower mode; it is provided modelling with splines a function like:

$$
v_{k}=f\left(\Delta v, v_{k-1}, d_{k-1}\right)
$$

where $\Delta v$ is the speed difference between follower and leader, estimated with Kalmann filter and $d_{k-1}$ the current distance from the frontal vehicle reported by sensors.

\section{CONClusion}

In this paper an autonomous vehicle pathplanner based on potential fields is presented. The most important feature of the controller consists in limiting the evaluation of trajectories, generating only candidates that the underlying control system can perform. The use of cost maps allows fusion of information provided by several sensors in a very simple and effective way providing fast assessment of the trajectories reliability. In such way the planner is capable of generating and following a trajectory in real-time.

During development several tests were performed, involving a numbers of kilometers of autonomous driving in different weather and environmental conditions. Experimental results shown in this paper were obtained by testing the vehicles inside Parma University Campus (Figure 10), a $2 \mathrm{~km}$ long loop repeated several times in autonomous driving, and in the surroundings of Parma either in urban and rural scenarios.

In figures $11 \mathrm{a}$ and $11 \mathrm{~b}$ the results of WayPoint Following autonomous tests ( 6 laps performed inside the campus) 


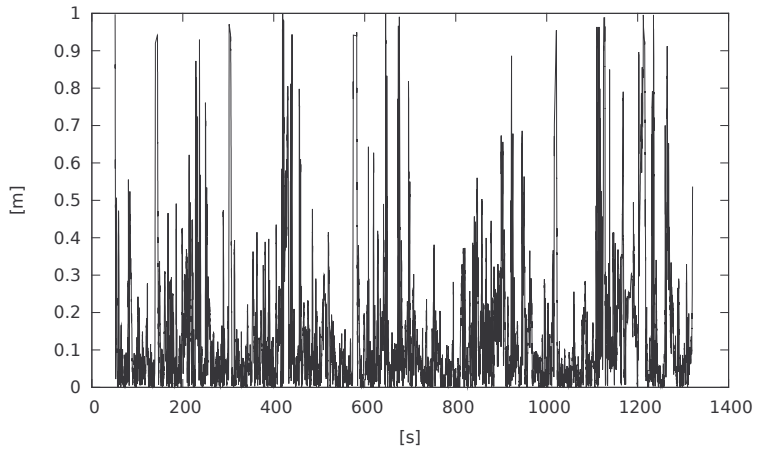

(a)

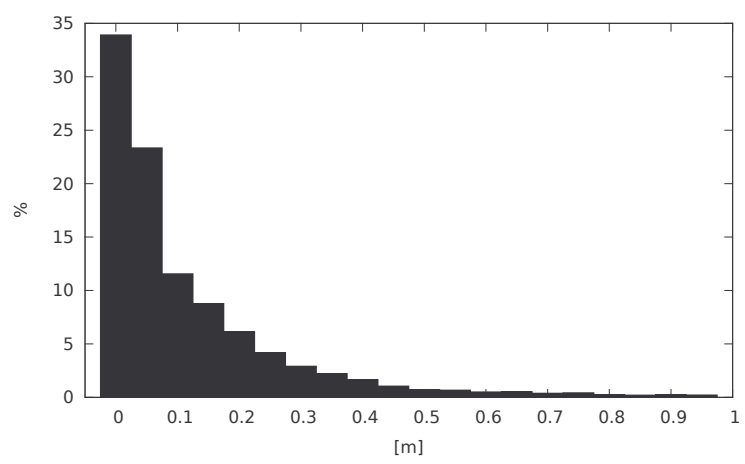

(b)

Fig. 11. Plot of lateral crosstrack error $(a)$ and relative histogram $(b)$ during the experiment of 22 minutes of autonomous operations on campus with autonomous GPS Waypoint following.

are presented: the mean crosstrack error is $13 \mathrm{~cm}$, and its standard deviation is $15 \mathrm{~cm}$. The average speed on this test was $26 \mathrm{~km} / \mathrm{h}$ (maximum $46 \mathrm{~km} / \mathrm{h}$ ).

Autonomous driving performance of leader follower capability in urban and extraurban areas are shown in figures 12a and $12 \mathrm{~b}$ : the mean crosstrack error is $17 \mathrm{~cm}$, and its standard deviation is $18 \mathrm{~cm}$. The average speed on this test was $28 \mathrm{~km} / \mathrm{h}$ (maximum $50 \mathrm{~km} / \mathrm{h}$ ).

A significant increase in the lateral crosstrack error is caused by the presence of obstacles along the road: the potential generated by obstacles influences the trajectory performed by the vehicle, moving it away from the WayPoint trajectory.

The controller has been validated experimentally on the vehicles taking part in the VIAC experiment and demonstrated good capabilities to support our cross-Asia trip.

\section{ACKNOWLEDGMENTS}

The work described in this paper has been developed in the framework of the Open intelligent systems for Future Autonomous Vehicles (OFAV) Project funded by the European Research Council (ERC) within an Advanced Investigators Grant.

\section{REFERENCES}

[1] A. Piazzi, C. Guarino Lo Bianco, M. Bertozzi, A. Fascioli, and A. Broggi, "Quintic $\mathrm{G}^{2}$-splines for the Iterative Steering of Visionbased Autonomous Vehicles," IEEE Trans. on Intelligent Transportation Systems, vol. 3, no. 1, pp. 27-36, Mar. 2002.

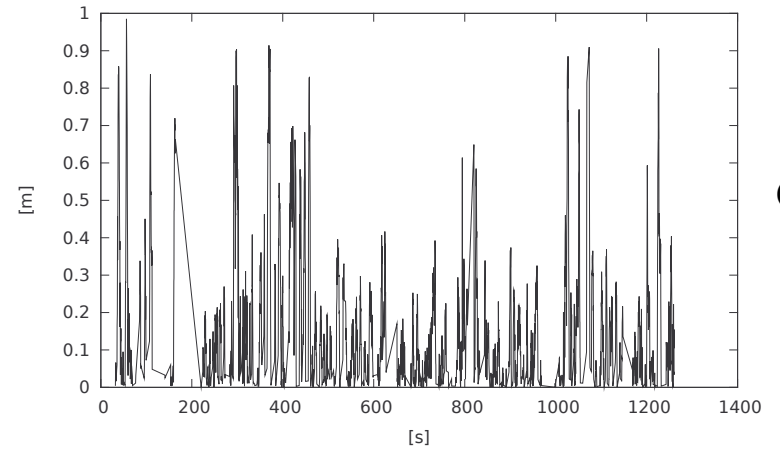

(a)

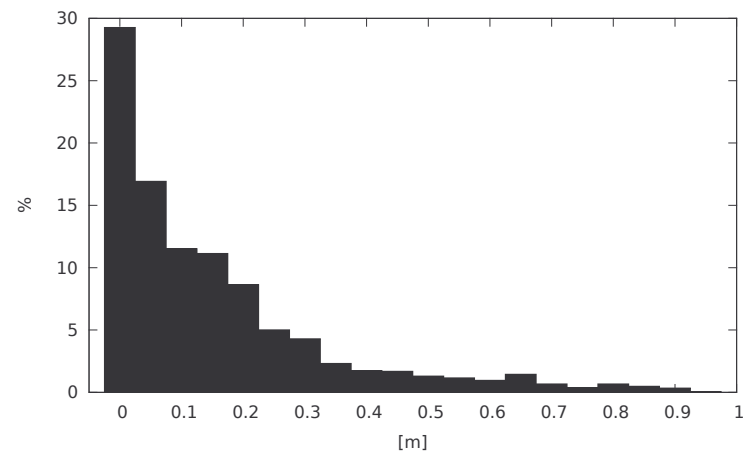

(b)

Fig. 12. Plot of lateral crosstrack error $(a)$ and relative histogram $(b)$ during the experiment of 21 minutes of autonomous operations in urban and rural environments with vehicle in autonomous Leader Vehicle following.

[2] S. Thrun, M. Montemerlo, H. Dahlkamp, D. Stavens, A. Aron, J. Diebel, P. Fong, J. Gale, M. Halpenny, G. Hoffmann, K. Lau, C. Oakley, M. Palatucci, V. Pratt, P. Stang, S. Strohband, C. Dupont, L.-E. Jendrossek, C. Koelen, C. Markey, C. Rummel, J. van Niekerk, E. Jensen, P. Alessandrini, G. Bradski, B. Davies, S. Ettinger, A. Kaehler, A. Nefian, and P. Mahoney, "Winning the darpa grand challenge," Journal of Field Robotics, 2006, accepted for publication.

[3] Y.-L. Chen, V. Sundareswaran, C. Anderson, A. Broggi, P. Grisleri, P. P. Porta, P. Zani, and J. Beck, "Terramax ${ }^{\mathrm{TM}}$ : Team oshkosh urban robot," J. Field Robot., vol. 25, no. 10, pp. 841-860, 2008.

[4] M. Linderoth, K. Soltesz, and R. Murray, "Nonlinear lateral control strategy for nonholonomic vehicles," in 2008 American Control Conference, Seattle, USA, June 2008.

[5] G. Hoffmann, C. Tomlin, M. Montemerlo, and S. Thrun, "Autonomous automobile trajectory tracking for off-road driving: Controller design, experimental validation and racing," in American Control Conference, 2007. ACC '07, july 2007, pp. $2296-2301$.

[6] C. Urmson, J. Anhalt, H. Bae, J. D. Bagnell, C. Baker, R. E. Bittner, T. Brown, M. N. Clark, M. Darms, D. Demitrish, J. Dolan, D. Duggins, D. Ferguson, T. Galatali, C. M. Geyer, M. Gittleman, S. Harbaugh, M. Hebert, T. Howard, S. Kolski, M. Likhachev, B. Litkouhi, A. Kelly, M. McNaughton, N. Miller, J. Nickolaou, K. Peterson, B. Pilnick, R. Rajkumar, P. Rybski, V. Sadekar, B. Salesky, Y.-W. Seo, S. Singh, J. M. Snider, J. C. Struble, A. T. Stentz, M. Taylor, W. R. L. Whittaker, Z. Wolkowicki, W. Zhang, and J. Ziglar, "Autonomous driving in urban environments: Boss and the urban challenge," Journal of Field Robotics Special Issue on the 2007 DARPA Urban Challenge, Part I, vol. 25, no. 8, pp. 425-466, June 2008.

[7] T. Howard and A. Kelly, "Optimal rough terrain trajectory generation for wheeled mobile robots," International Journal of Robotics Research, vol. 26, no. 1, pp. 141-166, February 2007.

[8] Y. Kuwata, J. Teo, G. Fiore, S. Karaman, E. Frazzoli, and J. How, "Realtime motion planning with applications to autonomous urban driving," IEEE Trans. on Control Systems Technologies, vol. 17, no. 5, pp. 11051118, 2009.

[9] J. C. Gerdes and E. J. Rossetter, "A unified approach to driver assistance systems based on artificial potential fields," Journal of Dynamic Systems, Measurement, and Control, vol. 123, no. 3, pp. 431-438, 2001. 